10:30 Ам Topic: Catheter-Delivered Devices Are Preferred Over Surgery for Management of Secundum ASD

Moderator: Constantine Mavroudis

Pro: Andrew Redington

Con: Richard A. Jonas

11:15 AM Topic: The Symptomatic Infant Less Than 6 Months of Age With Tetralogy Should Be Managed With a Shunt

Moderator: William G. Williams

Pro: Charles D. Fraser

Con: Ross M. Ungerleider

12:00 NOON ADJOURN

\title{
The American Association for Thoracic Surgery Graham Traveling Fellowship, 2005-2006
}

\section{STIPEND \$75,000}

$\mathrm{T}$ The American Association for Thoracic Surgery Evarts A. Graham Memorial Traveling Fellowship was established in 1951 by The American Association for Thoracic Surgery. Administered through the Graham Educational Research Foundation Fellowship grants support study by young cardiothoracic surgeons from outside North America at sites of their choice within North America and include travel between sites. Goals of the program are to broaden overall training and increase international contacts. Each Fellow should plan to have one primary center for his/her activities where he/she should spend from six to nine months. The Fellow should have identified an individual at that center who will act as his/her sponsor and assist in planning the course of study at the host institution and at other institutions. In addition to the primary center, the Fellow is encouraged to plan to spend shorter periods of time at other secondary centers to enhance the Fellowship experience.

Awards are made to surgeons of unique promise who have been regarded as having the potential for later international thoracic surgical leadership. Since the inception of the Graham Fellowship, 51 young surgeons from 28 countries have completed the Fellowship.

The Fellowship provides a stipend of $\$ 75,000$. The Fellowship also provides two round-trip coach air fares from the recipient's country to his/her primary center. The dates of the beginning of the Fellowship year should be scheduled to coincide with the Annual Meeting of The American Association for Thoracic Surgery in San Francisco in April 2005. The Fellow will be introduced to the AATS membership at this meeting.
The Fellow should become involved predominantly with observation, consultation, teaching, and research at a variety of thoracic surgical training centers during the twelve months. Patient contact will be determined by the sponsoring surgeon and will conform to the regulations and licensing requirements of the state, province, or country in which he/she is studying. A candidate should have completed his/her formal training in general surgery and in thoracic and cardiovascular surgery, but he/she should not have reached a senior position. Candidates must be sufficiently proficient in English to realize the full benefits of the Fellowship. Candidates should not have received significant training in North America prior to making application for this award.

Applications for the Fellowship are available from:

Irving L. Kron, MD, President

Graham Education and Research Foundation

900 Cummings Center

Suite 221-U

Beverly, MA 01915

USA

Telephone 978-927-8330; fax 978-524-0461

For additional information and to download an application, please visit the AATS Web site at: www.aats.org/academic/ fellowships.

Completed applications must be returned no later than July 1, 2004. The selected candidate will be notified by December 15, 2004. 


\section{Evarts A. Graham Memorial Traveling Fellows}

\begin{tabular}{|c|c|c|}
\hline lst & $1951-52$ & L. L. Whytehead, MD, FRCS \\
\hline 2nd & $1953-54$ & W. B. Ferguson, MB, FRCS \\
\hline & & Newcastle-upon-Tyne, England \\
\hline $3 r d$ & $1954-55$ & $\begin{array}{l}\text { Lance L. Bromley, MChir, FRCS } \\
\text { London. England }\end{array}$ \\
\hline 4th & $1955-56$ & Raymond L. Hurt, FRCS \\
\hline & & Radlett Herts, England \\
\hline 5 th & $1956-57$ & Mathias Paneth, FRCS \\
\hline & & London, England \\
\hline 6 th & $1957-58$ & $\begin{array}{l}\text { Peter L. Brunnen, FRCS } \\
\text { Aberdeen, Scotland }\end{array}$ \\
\hline 7th & $1958-59$ & N G. Meyne, MD \\
\hline & & Amsterdam, The Netherlands \\
\hline 8th & $1960-61$ & Godrej S. Karai, MD \\
\hline & & Calcutta, India \\
\hline 9th & $1961-62$ & Fritz Helmer, MD \\
\hline & & Vienna, Austria \\
\hline 10th & $1962-63$ & Theodor M. Scheinin, MD \\
\hline & & Oulu, Finland \\
\hline 1lth & $1963-64$ & Masahiro Saigusa, MD \\
\hline & & Tokyo, Japan \\
\hline 12 th & 1963-64 & Adar J. Hallen, MD \\
\hline & & Uppsala, Sweden \\
\hline 13th & $1964-65$ & Stuart C. Lennox, MD \\
\hline & & London, England \\
\hline 14 th & $1964-65$ & Elias Carapistolis, MD, FACS \\
\hline & & Thessaloniki, Greece \\
\hline 15th & $1965-66$ & Gerhard Friehs, MD \\
\hline & & Graz, Austria \\
\hline 16th & $1965-66$ & Ary Blesovsky, MD \\
\hline & & London, England \\
\hline 17 th & $1966-67$ & C. Peter Clarke, FRACS \\
\hline & & Fitzroy, Australia \\
\hline 18th & $1966-67$ & G. B. Parulkar, MD \\
\hline & & Bombay, India \\
\hline 37 th & $1987-88$ & Byung-Chul Chang, MD \\
\hline & & Seoul, Korea \\
\hline 38th & $1988-89$ & Wang Cheng, MD \\
\hline & & Wuhan, The People's Republic of China \\
\hline 39 th & $1989-90$ & Christopher J. Knott-Craig, MD \\
\hline & & Cape Town, Republic of South Africa \\
\hline 40th & $1991-92$ & Ko Bando, MD \\
\hline & & Okayama, Japan \\
\hline $41 \mathrm{st}$ & $1992-93$ & Timothy E. Oaks, MD \\
\hline & & Hershey, Pennsylvania, USA \\
\hline 42nd & 1993-94 & Alain E. Serraf, MD \\
\hline & & Creteil, France \\
\hline 43rd & $1995-96$ & Cornelius McKown Dyke, MD \\
\hline & & Richmond, Va, USA \\
\hline 44th & $1996-97$ & Monica Robotin-Johnson, MD \\
\hline & & Sydney, Australia \\
\hline
\end{tabular}

\begin{tabular}{|c|c|c|}
\hline 19th & $1967-68$ & Claus Jessen, MD \\
\hline 20th & $1969-70$ & Peter Bruecke, MD \\
\hline & & Linz-Puchenau, Austria \\
\hline $21 \mathrm{st}$ & $1970-71$ & Michel S. Slim, MD \\
\hline & & Beirut, Lebanon \\
\hline 22nd & $1971-72$ & Severi Pellervo Mattila, MD \\
\hline & & Helsinki, Finland \\
\hline $23 r d$ & $1972-73$ & Yasuyuki Fujiwara, MD \\
\hline & & Tokyo, Japan \\
\hline 24th & $1973-74$ & Marc Roger de Leval, MD \\
\hline & & London, England \\
\hline 25 th & $1974-75$ & J. J. DeWet Lubbe, MD \\
\hline & & Bellville, Republic of South Africa \\
\hline 26th & $1975-76$ & Mieczyslaw Trenkner, MD \\
\hline & & Gdansk, Poland \\
\hline 27 th & $1976-77$ & Bum Koo Cho, MD \\
\hline & & Seoul, Korea \\
\hline 28th & $1977-78$ & Alan William Gale, MD, FRACP, FRACS \\
\hline & & Sydney, Australia \\
\hline 29th & $1978-79$ & Eduardo Otero Coto, MD \\
\hline & & Valencia, Spain \\
\hline 30 th & $1980-81$ & Richard Firmin, MD \\
\hline & & London, England \\
\hline $31 \mathrm{st}$ & $1981-82$ & Claudio A. Salles, MD \\
\hline & & Belo Horizonte, Brazil \\
\hline 32nd & $1982-83$ & Yasuhisa Shimazaki, MD \\
\hline & & Osaka, Japan \\
\hline 33rd & $1983-84$ & Georg S. Kobinia, MD \\
\hline & & Linz, Austria \\
\hline 34 th & $1984-85$ & Aram Smolinsky, MD \\
\hline & & Tel Hashomer, Israel \\
\hline 35 th & $1985-86$ & Florentino J. Vargas, MD \\
\hline & & Buenos Aires, Argentina \\
\hline 36 th & $1986-87$ & Ari L. J. Harjula, MD \\
\hline & & Helsinki, Finland \\
\hline 45 th & $1997-98$ & Jun Wang, MD \\
\hline & & Beijing, China \\
\hline 46th & 1998-99 & Christian Kreutzer, MD \\
\hline & & Buenos Aires, Argentina \\
\hline 47 th & $1999-2000$ & Anders Franco-Cereceda, MD \\
\hline & & Stockholm, Sweden \\
\hline 48th & $2000-01$ & Albertus Scheule, MD \\
\hline & & Tuebingen, Germany \\
\hline 49th & 2001-02 & Anna Maria Ciccone, MD \\
\hline & & Rome, Italy \\
\hline 50 th & $2002-03$ & Cliff K. C. Choong, MD \\
\hline & & Auckland, New Zealand \\
\hline $51 \mathrm{st}$ & 2003-04 & Edvin Prifti, MD \\
\hline & & Tirana, Albania \\
\hline 52nd & 2004-05 & Smruti Ranjan Mohanty, MD \\
\hline & & Bangalore, Karnataka, India \\
\hline
\end{tabular}

